\title{
Spontaneous CPT Violation and Quantum Anomalies in a Model for Matter-Antimatter Asymmetry in the Cosmos $t$
}

\author{
Nick E. Mavromatos * (D) and Sarben Sarkar \\ Theoretical Particle Physics and Cosmology Group, Department of Physics, King's College London, Strand, \\ London WC2R 2LS, UK; sarben.sarkar@kcl.ac.uk \\ * Correspondence: Nikolaos.Mavromatos@kcl.ac.uk; Tel.: +44-207-848-2168 \\ † This paper is based on the talk at the 7th International Conference on New Frontiers in Physics (ICNFP 2018), \\ Crete, Greece, 4-12 July 2018.
}

Received: 27 November 2018; Accepted: 20 December 2018; Published: 25 December 2018

\begin{abstract}
We review scenarios of baryogenesis through leptogenesis at early epochs of the universe, in string-inspired minimal extensions of the Standard Model (SM), involving heavy right-handed Majorana neutrinos. Spontaneous violation of CPT symmetry is induced by appropriate (in general, temperature-dependent) backgrounds of the Kalb-Ramond (KR) axion field, which has its origins in the (bosonic) massless string multiplet. As interesting features of the model, we also discuss two issues associated with quantum (chiral) anomalies: (i) the non-contribution of the KR axion background to the (anomalous) chiral magnetic effect, which arises in the presence of external electromagnetic fields and non-zero chiral chemical potentials of charged fermions; and (ii) the potential role of quantum fluctuations of the KR axion on the (anomalous) radiative generation of a Majorana mass for the right-handed neutrinos themselves.
\end{abstract}

Keywords: leptogenesis; CPT violation; string effective theories

\section{Introduction}

It is well known [1-3] that the Standard Model (SM) of particle physics cannot explain the observed (primarily baryonic) matter-antimatter asymmetry in the universe $[4,5]$, which requires

$$
\Delta n(T \sim 1 \mathrm{GeV})=\frac{n_{B}-n_{\bar{B}}}{n_{B}+n_{\bar{B}}} \sim \frac{n_{B}-n_{\bar{B}}}{s}=(8.4-8.9) \times 10^{-11}
$$

for (cosmic) times $t \sim 10^{-6} \mathrm{~s}$ and temperatures $T \sim 1 \mathrm{GeV}$. In the above formula, $n_{B}\left(n_{\bar{B}}\right)$ denotes the (anti-)baryon density in the universe, and $s$ is the entropy density of the universe, scaling with the cubic power of the temperature. The observation of charge-parity violation led Sakharov to conjecture that fundamental particle interactions [6] could lead to Baryon Asymmetry provided: (i) there was Baryon (B) number violation; (ii) there was Charge (C) and Charge-Parity (CP) symmetry breaking; and (iii) the universe was out of chemical equilibrium (so that the asymmetry between matter and antimatter is not washed out). Sakharov assumed that CPT is a fundamental and unbroken symmetry (where T denotes time-reversal symmetry). Although the Sakharov conditions are satisfied qualitatively in the $\mathrm{SM}$, the calculated baryon asymmetry in the universe (BAU) is found to be several orders of magnitude smaller than the observed one (Equation (1)).

There are two types of non-equilibrium processes in the early universe that can produce asymmetries between particles and antiparticles: the first type concerns processes generating 
asymmetries between leptons and antileptons (leptogenesis) [7-11], while the second produces asymmetries between baryons and antibaryons directly (baryogenesis) [12-15].

Several non-trivial extensions of the SM exist, including supersymmetric and (super)string theories and extra dimensional models, which involve extra sources for $\mathrm{CP}$ violation so that they could reproduce the observed asymmetry (Equation (1)), in accordance to Sakharov's conditions. None of the ingredients of those models, however, have been verified by experiment to date. Moreover, fine tuning and some ad hoc assumptions are involved in such scenarios, especially in connection with the magnitude of the $C P$ violating phases and the associated decay widths. Consequently, the quest for a proper understanding of the observed BAU is incomplete and requires further investigation of physics beyond the SM.

Some of the attempts mentioned above involve the elegant mechanism of baryogenesis via leptogenesis. In such scenarios, a lepton asymmetry is generated first, by means of decays of right handed sterile neutrinos to SM particles, and is subsequently communicated to the baryon sector by means of sphaleron processes which violate both Baryon (B) and Lepton (L) numbers, but preserve the difference B-L [16-22].

Heavy sterile neutrinos, through the the seesaw mechanism [23-27], play another essential role in particle physics, since they provide a natural explanation for the smallness of the mass of the three light neutrinos in the SM, as suggested by the observed neutrino oscillations [28,29]. A model that extends the SM in such a way as to understand both neutrino oscillations and BAU would be economical and attractive.

All the above scenarios for the generation of BAU respect the CPT theorem [30]: relativistic, local and unitary Lagrangians (without gravity) are invariant under the combined action of $\mathrm{C}, \mathrm{P}$ and $\mathrm{T}$ transformations [31-36]. Such a theorem is a cornerstone of modern particle physics and is the reason that it was assumed in the scenario of Sakharov [6]. It is still possible that CPT is spontaneously broken. In addition, some of the assumptions in the proof of the CPT theorem may not necessarily hold in the early universe when quantum gravity effects can be important. If there is CPT violation (CPTV), the necessity of non-equilibrium processes for the generation of BAU in $C P T$ invariant theories may be relaxed.

In our previous work [37-41], we considered Lorentz invariance violating (LV) backgrounds in the early universe as a form of spontaneous violation of Lorentz and CPT symmetry. If LV is the primary source of CPTV, then the latter can be studied within a local effective field theory framework, which is known as the Standard Model Extension (SME) [42]. From experiments, there are very stringent upper bounds on the magnitude of the parameters determining Lorentz and CPT violation in the SME [43-46]. However, under the extreme conditions of the very early universe, such violations could be significantly stronger than in the present era. Here, we determine relaxation mechanisms (with temperature), by means of which such strong CPTV at early eras diminishes to phenomenologically acceptable values today.

Within the SME framework, there have been suggestions [47] of direct baryogenesis due to LV and CPTV terms in the the effective action, which induce "effective chemical potentials", e.g., for quarks. In the presence of a chemical potential, the populations of quarks and antiquarks are already different within thermal equilibrium, since the the particle and antiparticle phase-space distribution functions $f(E, \mu), f(\bar{E}, \bar{\mu})$, with $E$ the energy are different. All these cause a difference in the corresponding equilibrium populations

$$
\left.f(E, \mu)=[\exp (E-\mu) / T) \pm 1]^{-1}, \quad f(\bar{E}, \bar{\mu})=[\exp (\bar{E}-\bar{\mu}) / T) \pm 1\right]^{-1},
$$

where $+(-)$ denotes a fermionic (bosonic) particle species and a bar over a quantity associates the quantity with an antiparticle; we have for the chemical potentials $\bar{\mu}=-\mu$. In principle, such scenarios, in the SME context, can lead to alternative explanations for the observed matter-antimatter asymmetry, provided that detailed mechanisms for freeze-out of particle interactions are provided. However, 
in [47], microscopic models leading to such SME Lagrangians and related phenomena have not been provided. ${ }^{1}$

In our papers [37-41], we embarked on a study of CPTV induced matter-antimatter asymmetry in the universe, with a microscopic ultra-violet complete string theory model in mind. Within the framework of effective field theories, the string theory model can be mapped into specific SME models. In particular, we consider minimal extensions of the SM, involving massive right handed (sterile) Majorana neutrinos (RHN) with a Higgs portal, connecting the RHN sector to the SM one. The RHN couple to the massless Kalb-Ramond (KR) pseudoscalar field (KR axion), dual to the spin-one antisymmetric tensor field of the massless bosonic string multiplet [50,51]. The decays of the RHN into SM leptons, in the presence of LV and CPTV backgrounds of the KR axion field, lead to leptogenesis; subsequently, baryogenesis ensues through B-L conserving sphaleron processes in the SM sector.

The structure of the article is as follows: in Section 2. we review the basic features of the mechanism for the CPTV-induced lepton asymmetry in a rather generic framework, without specifying the microscopic origin of the CPTV. In Section 3, we discuss microscopic scenarios for such a CPTV-induced matter-antimatter asymmetry, within the framework of effective field theories representing the low-energy limit of strings. In Section 4, we discuss the role of the CPTV background on the chiral magnetic effect (CME), which has its origin in the chiral anomaly; CME characterises charged fermion systems such as quarks (effectively massless at sufficiently high temperature) in the presence of external magnetic fields. As we demonstrate, the CPTV KR axion background does not participate in the effect. In Section 5, we discuss scenarios in which the quantum fluctuations of the KR axion can lead to radiative (anomalous) generation of the RHN mass, which go beyond the standard seesaw mechanisms. Finally, Section 6 contains our conclusions and outlook for future work.

\section{Spontaneous-CPT-Violation-Induced Leptogenesis}

In Refs. [37-40], we discussed the generation of matter-antimatter asymmetry in the universe (in particular baryogenesis via leptogenesis) by invoking spontaneous breaking of CPT symmetry in the early universe through appropriate backgrounds. In particular, in [39,40], we considered leptogenesis originating from tree-level decays of RHN into SM leptons, in the presence of generic CPTV time-like axial backgrounds. In the cosmological (i.e., Robertson-Walker) frame of the early universe, the background is assumed constant. The relevant Lagrangian is given by:

$$
\mathcal{L}=\mathcal{L}_{\mathrm{SM}}+i \bar{N} \not N-\frac{m_{N}}{2}\left(\overline{N^{c}} N+\bar{N} N^{c}\right)-\bar{N} B \gamma^{5} N-\sum_{k} y_{k} \bar{L}_{k} \tilde{\varphi} N+\text { h.c. }
$$

where $\mathcal{L}_{\mathrm{SM}}$ denotes the SM Lagrangian, $B_{\mu}$ is a CPTV background field, associated with physics beyond the SM, $N$ is the RHN field, of (Majorana) mass $m_{N}, \tilde{\varphi}$ is the adjoint $\left(\tilde{\varphi}_{i}=\varepsilon_{i j} \varphi_{j}\right)$ of the Higgs field $\varphi$, and $L_{k}$ is a lepton (doublet) field of the SM sector, with $k$ a generation index. $y_{k}$ is a Yukawa coupling, which is non-zero and provides a non-trivial (Higgs portal) interaction between the RHN and the SM sectors. In the case of $[39,40]$, a single sterile neutrino species suffices to generate phenomenologically relevant lepton asymmetry; in addition, from now on, for simplicity, we restrict ourselves to the first generation $(k=1)$ of SM leptons, and set

$$
y_{1}=y
$$

1 If the effects of CPTV manifested themselves only in mass differences between particles and antiparticles, then, under the natural assumptions [48], it has been argued that the dominant effects should come from the quarks; since the quark-antiquark mass differences are bounded from above by the current bounds on the proton-antiproton mass difference [49], the induced BAU, due to the corresponding differences in the thermal distribution functions (Equation (2)), turns out to be several orders of magnitude smaller than the observed value (Equation (1)). This result is reached [48] by applying the standard linear scaling of the quark mass with temperature in the range of validity of (1). 
In the scenario of $[39,40]$, the CPTV background $B_{\mu}$ is assumed to have only a non-zero temporal component, which is taken to be constant in the Robertson-Walker frame of the early universe,

$$
B_{0}=\text { const } \neq 0, B_{i}=0, i=1,2,3 .
$$

In this case, the Lagrangian (Equation (3)) assumes the form of a SME Lagrangian in a Lorentz and CPTV background [42].

A lepton asymmetry is then generated due to the CP and CPTV tree-level decays of the RHN into SM leptons, in the presence of the background (Equation (5)), induced by the Higgs portal Yukawa interactions of (Equation (3)) [39,40]:

$$
\begin{aligned}
\text { Channel I : } & N \rightarrow l^{-} h^{+}, v h^{0}, \\
\text { Channel II }: & N \rightarrow l^{+} h^{-}, \bar{v} h^{0} .
\end{aligned}
$$

where $\ell^{ \pm}$are charged leptons, $v(\bar{v})$ are light, "active", neutrinos (antineutrinos) in the SM sector, $h^{0}$ is the neutral Higgs field, and $h^{ \pm}$are the charged Higgs fields ${ }^{2}$. As a result of the $B_{0} \neq 0$ background (Equation (5)), the decay rates of the RHN between the channels I and II are different, resulting in a lepton asymmetry, $\Delta L^{T O T}$, which then freezes out at a temperature $T_{D}$. In [40], a detailed study of the associated Boltzmann equations for the processes in (Equation (6)), and the reciprocal processes, has led to the result ${ }^{3}$ :

$$
\frac{\Delta L^{T O T}}{s} \simeq(0.016,0.019) \frac{B_{0}}{m_{N}}, \quad \text { at freezeout temperature } \quad T=T_{D}: \quad m_{N} / T_{D} \simeq(1.44,1.77) .
$$

This implies that phenomenologically acceptable values of the lepton asymmetry of $\mathcal{O}\left(8 \times 10^{-11}\right)$, which can then be communicated to the baryon sector via Baryon-minus-Lepton-number $(B-L)$ conserving sphaleron processes in the SM, thus producing the observed amount of baryon asymmetry (baryogenesis) in the universe, occur for values of

$$
\frac{B_{0}}{m_{N}} \sim 10^{-9}, \quad \text { at freezeout temperature } T=T_{D}: \quad m_{N} / T_{D} \simeq(1.77,1.44),
$$

The different values $(a, b)$ of the numerical coefficients in the right-hand-side of the two equations in Equation (7), are due to two different analytical methods (series expansion (a) and integrating factor (b) method [40], respectively) used in the Padè approximate solution of the Boltzmann equations associated with Equation (6). With value of the Yukawa coupling (Equation (4)) $y \sim 10^{-5}$, and for $m_{N}=\mathcal{O}(100) \mathrm{TeV}[39,40]$, we thus obtain a $B_{0} \sim 0.1 \mathrm{MeV}$, for phenomenologically relevant leptogenesis to occur at $T_{D} \simeq(56-69) \mathrm{TeV}$, in our scenario. In $[39,40]$, the microscopic nature of the background $B_{0}$ is not discussed in detail.

\section{Microscopic String-Inspired Models}

The field strength of the spin- 1 antisymmetric tensor (Kalb-Ramond (KR)) field of the massless (bosonic) gravitational multiplet of strings [37-39] could provide a simple and physically interesting CPTV background that plays the role of $B_{0}$. In the closed-string sector the massless bosonic gravitational multiplet of a string theory consists of three fields [50,51]: a traceless, symmetric, spin-2 tensor field $g_{\mu \nu}$ identified with the graviton; a spin 0 (scalar) field, the dilaton $\Phi$, identified with the trace of the

2 At high temperatures, above the spontaneous electroweak symmetry breaking, the charged Higgs fields $h^{ \pm}$do not decouple from the physical spectrum, and play an important role in leptogenesis.

3 There is a certain uncertainty related to the Taylor expansions used in our Padé analysis and to indicate this a range of numbers in brackets is given below. 
graviton; and the spin- 1 antisymmetric tensor (Kalb-Ramond) field $B_{\mu v}=-B_{v \mu}$. In the closed string sector, there is a $U(1)$ gauge symmetry $B_{\mu v} \rightarrow B_{\mu v}+\partial_{\mu} \theta_{v}-\partial_{\nu} \theta_{\mu}$, which characterises the target-space effective action. This implies that the latter depends only on the field strength of the field $B_{\mu v}$, which is a three-form with components

$$
H_{\mu v \rho}=\partial_{[\mu} B_{v \rho]},
$$

where the symbol $[\ldots]$ denotes complete antisymmetrisation of the respective indices. The three-form $H_{\mu v \rho}$ satisfies the Bianchi identity

$$
\partial_{[\mu} H_{v \rho \sigma]}=0,
$$

by construction ${ }^{4}$.

In the Einstein frame [52-54], the bosonic part of the (four-space-time-dimensional) effective action, $S_{B}$ reads:

$$
S_{B}=\frac{1}{2 \kappa^{2}} \int d^{4} x \sqrt{-g}\left(R-e^{-4 \Phi} H_{\lambda \mu \nu} H^{\lambda \mu v}-\Omega\right)+\ldots
$$

where $\kappa^{2}=8 \pi G$, and $G=M_{P}^{-2}$ is the $(3+1)$-dimensional Newton constant (with $M_{P}=1.22 \times$ $10^{19} \mathrm{GeV}$, the four-dimensional Planck mass). $M_{P}$ is related to the string mass scale $M_{S}$ via [50,51]: $G^{-1}=\mathcal{V}^{(n)} M_{s}^{2+n}$, with $\mathcal{V}^{(n)}$ a compactification volume (or appropriate bulk volume factor, in brane universe scenarios). For standard (ten-space-time dimensional) superstrings, $n=6$. The $\Omega$ in Equation (11) represents a vacuum energy term. It arises in non-critical-dimension string models [55], or from bulk contributions in brane universe scenarios; in the latter case, contributions can be of anti-de-Sitter-type $[56,57]$. The $\ldots$ represent derivatives of the dilaton field, $\Phi$; these derivative terms are assumed to be small $[39,40]$ at the epoch of the universe relevant for leptogenesis; hence, we may approximate $\Phi \simeq$ constant, which can thus be absorbed in appropriate normalisations of the KR field. In this approximation, the vacuum energy term $\Omega$ is treated as a constant, to be determined phenomenologically by requiring appropriately suppressed vacuum energy contributions. We come back to this issue below.

It is known [50-54] that the KR field strength terms $H^{2}$ in (11) can be absorbed into a generalised curvature scheme with a "torsionful connection" $\bar{\Gamma}_{\mu v}^{\rho}$ given by $\bar{\Gamma}_{\mu v}^{\rho}=\Gamma_{\mu v}^{\rho}+H_{\mu v}^{\rho} \neq \bar{\Gamma}_{v \mu}^{\rho}$, where $\Gamma_{\mu \nu}^{\rho}=\Gamma_{v \mu}^{\rho}$ is the torsion-free Christoffel symbol; thus, the contorsion tensor is determined by the $H_{\mu v}^{\rho}$ field strength.

In our approach we include fermion fields, of mass $m$, as well. The contorsion interpretation of $H_{\mu \nu}^{\rho}$ implies a minimal coupling of this field to the axial fermion current, since the corresponding Dirac term for fermions in torsionful gravitational backgrounds reads $[39,40,58]$ :

$$
\begin{aligned}
S_{\text {Dirac }} & =\int d^{4} x \sqrt{-g}\left[\frac{\imath}{2}\left(\bar{\psi} \gamma^{\mu} \overline{\mathcal{D}}(\bar{\omega})_{\mu} \psi-\left(\overline{\mathcal{D}}(\bar{\omega})_{\mu} \bar{\psi}\right) \gamma^{\mu} \psi\right)-m \bar{\psi} \psi\right], \\
& =\int d^{4} x \sqrt{-g} \bar{\psi}\left(\imath \gamma^{\mu} \partial_{\mu}-m\right) \psi+\int d^{4} x \sqrt{-g}\left(\mathcal{F}_{\mu}+B_{\mu}\right) \bar{\psi} \gamma^{5} \gamma^{\mu} \psi, \\
\overline{\mathcal{D}}_{a} & =\partial_{a}-\frac{\imath}{4} \bar{\omega}_{b c a} \sigma^{b c}, \quad \sigma^{a b}=\frac{l}{2}\left[\gamma^{a}, \gamma^{b}\right], \\
\mathcal{F}^{\mu} & =\varepsilon^{a b c \mu} e_{b \lambda} \partial_{a} e_{c}^{\lambda}, \quad B^{\mu}=-\frac{1}{4} e^{-2 \phi} \varepsilon_{a b c}{ }^{\mu} H^{a b c}, \quad J^{5 \mu}=\bar{\psi} \gamma^{\mu} \gamma^{5} \psi,
\end{aligned}
$$

4 In (Heterotic) string theory, in the presence of gauge and gravitational fields, the right-hand-side of Equation (9) is modified by appropriate (parity-violating) Chern-Simons three-forms. The right-hand side of the Bianchi identity (Equation (10)) becomes non-zero, a sign of gauge and gravitational anomalies [50,51]. We do not deal explicitly with such (higher derivative) terms here, as they are not directly relevant to our leptogenesis scenario. We briefly demonstrate, however, in Section 4, that their inclusion does not affect the chiral magnetic effect. 
where $e_{\mu}^{a}(x)$ are the vielbeins, $g_{\mu v}(x)=e_{\mu}^{a}(x) \eta_{a b} e_{v}^{b}(x)$, with $\eta_{a b}$ the Minkowski metric of the tangent space at a space-time point with coordinates $x^{\mu}$. The generalised spin-connection is: $\bar{\omega}_{a b \mu}=\omega_{a b \mu}+$ $K_{a b \mu}, K_{a b c}=\frac{1}{2}\left(H_{c a b}-H_{a b c}-H_{b c a}\right)=-\frac{1}{2} H_{a b c}$, where, $\omega_{a b \mu}$ denotes the standard torsion-free spin connection; as usual, Latin letters denote tangent-space indices, while Greek letters refer to space-time indices. In Equation (12), we use standard properties of the $\gamma$-matrices. For a Robertson-Walker metric $g_{\mu \nu}$ background, of relevance to us here, $\mathcal{F}_{\mu}=0$, and thus we can write the action (Equation (12)) in the form:

$$
S_{\text {Dirac }}=\int d^{4} x \sqrt{-g} \bar{\psi}\left(\imath \gamma^{\mu} \partial_{\mu}-m\right) \psi+\int d^{4} x \sqrt{-g} B_{\mu} \bar{\psi} \gamma^{5} \gamma^{\mu} \psi \equiv S_{\text {Dirac }}^{\text {Free }}-\int d^{4} x \sqrt{-g} B_{\mu} J^{5 \mu},
$$

yielding a minimal coupling of the $H_{\mu v \rho}$ field to the fermion axial current.

In four space-time dimensions, the KR three-form $H$ can be expressed in terms of its dual pseudoscalar $b(x)(\mathrm{KR}$ "axion" ) field $[55,58]$

$$
\partial^{\mu} b=-\frac{1}{4} e^{-2 \phi} \varepsilon_{a b c}{ }^{\mu} H^{a b c},
$$

where $\varepsilon^{0123}=+1, \varepsilon_{0123}=-1$, etc. are the elements of the gravitationally covariant (totally antisymmetric) Levi-Civita tensor. On account of the definition of $B_{\mu}$ in Equation (12), this implies

$$
B^{\mu}=\partial^{\mu} b(x)
$$

The total (four-space-time-dimensional) effective action $S_{e f f}$, where we restrict our attention from now on, is given by the sum of the two actions $S_{B}$ and $S_{\text {Dirac }}$

$$
S_{\text {eff }}=S_{B}+S_{\text {Dirac }}
$$

and it can be expressed in terms of the KR axion field as follows [58]:

- $\quad$ First, we formulate the path integral, integrated over the KR field strength $H$.

- We insist on the preservation of the Bianchi identity (Equation (10)) at a quantum level, via the addition of appropriate counterterms (in a renormalisation group sense) order by order in perturbation theory. This guarantees the conservation of the "H-torsion charge" $Q=$ $\int d^{3} x \varepsilon_{i j k} H^{i j k}$, which is implemented in the path-integral via a $\delta$-functional constraint in the form $\delta\left(\kappa^{2} \varepsilon^{\mu v \rho \sigma} \partial_{\mu} H_{\nu \rho \sigma}\right)$, and expressing the latter in terms of a (dimensionless) Lagrange multiplier field $b(x)$, which eventually will correspond to the dual KR axion field:

$$
\begin{aligned}
\delta\left(\kappa^{2} \varepsilon^{\mu v \rho \sigma} \partial_{\mu} H_{\nu \rho \sigma}\right) & =\int \mathcal{D} b \exp \left[i \kappa^{-2} \int d^{4} x \sqrt{-g} b(x) \varepsilon_{\mu \nu \rho \sigma} \partial^{\mu} H^{v \rho \sigma}\right] \\
& =\int \mathcal{D} b \exp \left[-i \kappa^{-2} \int d^{4} x \sqrt{-g} \partial^{\mu} b(x) \varepsilon_{\mu \nu \rho \sigma} H^{v \rho \sigma}\right]
\end{aligned}
$$

where the second equality has been obtained by partial integration, upon assuming that the KR field strength dies out at spatial infinity.

- Integrating out the $H$-field in the path integral with the action in Equation (16), we obtain a path integral over the Lagrange multiplier field $b(x)$,

$$
\begin{aligned}
Z & =\int \mathcal{D} g \mathcal{D} \psi \mathcal{D} \bar{\psi} \mathcal{D} b \exp \left[{ }^{2} S_{\text {eff }}\right], \\
S_{\text {eff }} & \left.=\frac{1}{2 \kappa^{2}} \int d^{4} x \sqrt{-g}\left(R+\frac{8}{3} \partial_{\sigma} b \partial^{\sigma} b-\Omega\right)+S_{\text {Dirac }}^{\text {Free }}-\int d^{4} x \sqrt{-g} \partial_{\mu} b J^{5 \mu}-\frac{3 \kappa^{2}}{16} \int d^{4} x \sqrt{-g} J_{\mu}^{5} J^{5 \mu}\right] .
\end{aligned}
$$


In realistic situations, of relevance to us here, there are many fermion species $\psi_{i}$, with various masses $m_{i}, i=1,2, \ldots, N$. Then, the axial current is a sum over such species

$$
J_{\mu}^{5}=\bar{\psi}_{i} \gamma_{\mu} \gamma^{5} \psi_{i}
$$

where a repeated index $i$ denotes summation over $i$.

In the effective action $S_{\text {eff }}$ (Equation (18)), there is a four fermion axial-current-current term, which is a repulsive four-fermion term, yielding de-Sitter type (positive) contributions to the vacuum energy. Such positive contributions are standard in Einstein-Cartan theories of quantum torsion, where the torsion can be integrated exactly in a path integral.

On considering KR-axion backgrounds $\bar{b}(x)$ linear in cosmic time $t, \bar{b} \propto t$, for which $\dot{\bar{b}} \equiv B_{0}$ is constant (in the Robertson-Walker frame), and which are known to constitute exact backgrounds in bosonic non-critical strings [55], we observe that the $\partial \bar{b}-J^{5}$ interaction term in Equation (18) yields the CPT-Violating axial background $B_{0}$-term of the model discussed in $[39,40]$, which leads to leptogenesis. In this way, one obtains a microscopic origin of $B_{0}$ in the context of string-inspired models. We mention at this point that, as it turns out [39,40], the contributions of $B_{0}^{2}$ to the vacuum energy density, arising from the kinetic terms of the KR axion field, are too large during the leptogenesis era (since the $B_{0}$ required for phenomenologically acceptable lepton asymmetry is in the range of $0.1 \mathrm{MeV}(8)$ ). For the standard cosmology not to be affected significantly, one must require a fine tuning between $B_{0}^{2}$ and the bulk-induced vacuum energy density $\Omega$ (which receives anti-de-Sitter contributions from the bulk $[39,40])$, so that the total vacuum energy density acquires acceptably small values during the leptogenesis and subsequent eras.

A caveat to the above ideas is that $b$-axion backgrounds linear in time may not be exact solutions for superstrings in the presence of fermions. Moreover, even if they are, it is not known whether one could fine tune the associated parameters to guarantee a $B_{0}$ background (Equation (15)) in the $\mathrm{MeV}$ or lower range, as required for leptogenesis in the scenario of [39], given that a natural mass scale for such backgrounds is provided by the string scale itself $M_{S} \gg \mathrm{MeV}$ [55].

In $[39,40]$, an additional case for obtaining a CPTV KR axion background, corresponding to a constant $B_{0}=\dot{\bar{b}}$, which could lead to low $B_{0}$ appropriate for leptogenesis, was proposed, involving fermionic axial condensates, that have been conjectured to occur at the freezeout epoch of leptogenesis. Indeed, in the presence of fermions, the equations of motion for the KR background field $\bar{b}$ from Equation (18) imply:

$$
\partial_{\alpha}\left[\sqrt{-g}\left(\frac{8}{3 \kappa^{2}} \partial^{\alpha} \bar{b}-J^{5 \alpha}\right)\right]=0 .
$$

On assuming a (constant) temporal chiral condensate (which respects isotropy of the universe),

$$
0 \neq \text { const. }=\left\langle J^{05}\right\rangle=\left\langle\psi_{i}^{\dagger} \gamma^{5} \psi_{i}\right\rangle
$$

which may characterise fermions in the model except Majorana neutrinos [39], e.g., quarks in the SM sector, expanding the current in Equation (20) about the condensate (Equation (21)), $J_{0}^{5}=\left\langle J_{0}^{5}\right\rangle+$ quantum fluctuations, and ignoring the fluctuations, we then obtain from Equation (20)

$$
\partial_{t}\left[\sqrt{-g}\left(\frac{8}{3 \kappa^{2}} B^{0}-\left\langle J^{05}\right\rangle\right)\right]=0,
$$

which admits as a consistent solution (cf. (15))

$$
B^{0}=\dot{\bar{b}}=\frac{3 \kappa^{2}}{8}\left\langle J_{05}\right\rangle=\text { const. } \neq 0,
$$

implying a constant (in the Robertson-Wallker frame) Lorentz- and CPT-violating axial background $B^{0}$, as required for leptogenesis in the scenario of $[39,40]$. 
At this juncture, we make some remarks concerning the interpretation of the massless KR axion $b(x)$ field as a Goldstone boson of the associated spontaneously broken Lorentz symmetry. First, the breaking of Lorentz invariance is induced by the constant $B_{0}$ background solution in Equation (23), which has been argued to be an acceptable ground state (vacuum) of an underlying string theory model. The latter is fully Lorentz invariant, as is the effective action in Equation (18). This is the reason the breaking of Lorentz invariance associated with Equation (23) is considered as spontaneous, with the massless KR axion field $b(x)$ as the pertinent Goldstone Boson [55]. The breaking of Lorentz symmetry induces in turn a violation of CPT symmetry, given the general discussion in [36,59]. In the literature (based on quantum Yang-Baxter equations [33-35]), it has been argued that for spontaneously broken Lorentz symmetry the number of broken generators does not match the number of Goldstone bosons. In $[39,40]$, the spontaneous breaking of Lorentz invariance, induced by the constant- $\dot{b}$ background solution (Equation (23)), is associated with the formation of axial condensates among fermions ${ }^{5}$. In realistic models of phenomenological relevance, such condensates might arise dynamically, within the framework of chiral symmetry breaking scenarios in the early universe. In (the third reference of) [33], a discussion is presented on how, in the presence of chemical potential terms in the Lagrangian, a mass for Goldstone bosons could arise in the case of chiral symmetry breaking. At present, this latter issue needs further study: formally, the KR axion is massless in our scenario, and hence an approach such as that proposed in [33-35] might open a way for it to acquire mass. We hope to come back to a discussion of such important issues in the future.

In view of the LV and CPTV nature of $B_{0}$, it must satisfy the current-era stringent upper bounds, imposed by a plethora of precision measurements [43-46], according to which $\left|B_{0}\right|<0.01 \mathrm{eV}$ (with much more stringent constraints for spatial components $\left|B_{i}\right|<10^{-31} \mathrm{GeV}$ ). In the constant $B_{0}$ scenario of [39], this could be guaranteed, if one assumes that the chiral current condensate $\left\langle J^{05}\right\rangle$, is destroyed (due to unknown (beyond the SM) physics) at a temperature near the lepton-asymmetry freezeout $T \simeq T_{D} \simeq 10^{5} \mathrm{GeV}$. In that case, from Equation (22), upon taking into account a Robertson-Walker space-time, with scale factor $a(t) \sim T^{-1}$ at high temperatures, we obtain a cooling "law" for $B_{0} \sim T^{3}$, for $T \leq T_{D}$, which comfortably satisfies the above constraints in the current epoch [39], where the average temperature of the universe is that of the Cosmic Microwave Background $(\mathrm{CMB})$ radiation, $T_{0} \sim T_{\mathrm{CMB}} \simeq 0.23 \mathrm{meV}$. Indeed, with such a cooling law, taking into account that at decoupling $B_{0}\left(T_{D} \simeq 10^{5} \mathrm{GeV}\right)=\mathcal{O}(0.1 \mathrm{MeV})$, one finds [39]: $B_{0}\left(T_{0}\right)=\mathcal{O}\left(10^{-57}\right) \mathrm{GeV}$, which satisfies comfortably the bounds in [43-46]. Moreover, the corresponding vacuum energy density contributions, of order $B_{0}^{2}$, also satisfy the cosmological constraints [60].

Although appealing, the above scenario suffers from the fact that no concrete model has been proposed for the formation of the axial condensates. Since the four fermion axial interaction terms in Equation (18) are repulsive, they do not lead to condensate formation. Hence, one can invoke other mechanisms beyond the SM, e.g., through the appropriate exchange of heavy states that may exist in string theory models. However, such mechanisms have not been elaborated further in [40]. We are also currently agnostic as to the microscopic mechanism leading to the disappearance of the condensate soon after the freezeout; the $B_{0}$ background drops with the (cubic power of the) temperature, as time evolves [39,40], in order for the model to be compatible with the current stringent bounds on CPTV [42].

However, in [41], we demonstrated that one does not actually need the formation of axial condensates to obtain phenomenologically acceptable leptogenesis. In that work, by actually considering non-constant, temperature-dependent backgrounds $B_{0}$, obtained from the antisymmetric tensor field of string theory, scaling with the temperature as $T^{3}$, as described above, one can still produce a lepton asymmetry during the leptogenesis era via the decays of the right-handed neutrinos, with a phenomenology similar to the one in constant $B_{0}$ backgrounds $[39,40]$. An analytical

5 Par contrast, in [55], the Lorentz invariance violating constant background $B_{0}$ solution is argued to be an exact solution of bosonic string theory. 
approximation to the solution of the Boltzmann equations for that case, extending the study in [40], has been considered in [41]. The cubic dependence on temperature of the background $B_{0}(T) \sim T^{3}$, is dictated by the equation of motion of the KR-axion field (Equation (20)) in absence of an axial-fermion-current condensate, and is assumed all the way from temperatures around decoupling till the present day. Such a scaling is sufficiently mild, for the high temperature regime that we have considered, that the conditions for leptogenesis considered in [40] are only slightly modified. Leptogenesis still occurs at decoupling temperatures of order $T_{D} \simeq 100 \mathrm{TeV}$, where the background field $B_{0}=\mathcal{O}(\mathrm{keV})$ is smaller than the one predicted in $[39,40]$. Nonetheless, we obtain for the current-epoch the range of values $B_{0}\left(T_{0}\right)<3 \times 10^{-57} \mathrm{GeV}$. The upper bound is of the same order as found in the scenario of [39], and lies comfortably within the stringent current bounds of CPTV and LV [43-46] as well as the cosmological constraints on the vacuum energy density [60].

\section{KR axion Backgrounds, Anomalies and the Chiral Magnetic Effect}

In the presence of external magnetic fields, we briefly discuss potential effects of the axial torsion background $B_{0}$ on physical phenomena. Primordial magnetic fields are known to play a role in leptogenesis scenarios [61-64]. Specifically, we would be interested in examining whether $B_{0}$ plays any role in the so-called Chiral Magnetic Effect (CME) [65]. The CME has been conjectured to characterise systems (such as neutron stars or a hot QCD quark-gluon plasma (QG)) with external magnetic fields in the presence of a chiral chemical potential $\mu_{5}{ }^{6}$.

As we show, however, in our case, the CME will be unaffected by the presence of the axial KR background $B_{0}$, which in this respect plays a role analogous to an external axial vector potential that is known not to contribute to CME $[66,67]$. The non-contribution of the $B_{0}$ field to the CME in our case should also be expected from: (i) the fact that the phenomenon has its origin [65] in the chiral anomalies in quantum field theories $[68,69]$; (ii) the role of the $B_{0}$ field as a torsion in the low-energy string effective action; and (iii) the well-known result [70] that torsion contributions to the anomaly equation are removable by the addition of appropriate local counterterms (in a renormalisation group sense) to the corresponding effective action. Physical effects, such as the CME, should thus be free from such ambiguities. This result can also be understood from the fact that the chiral anomaly is associated with the index of the Dirac operator for fermions, which the torsion does not contribute to (as can be shown explicitly using heat kernel or other techniques [71]).

\subsection{Chiral Anomalies and the Chiral Magnetic Effect}

It will be instructive to first review briefly the CME phenomenon in the QG case [65]. Consider the $(3+1)$-dimensional flat space-time, finite-density massless quark Lagrangian $\mathcal{L}_{\text {quartks }}$ in the presence of a finite chemical potential $\mu$ and a finite chiral chemical potential $\mu_{5}$

$$
\mathcal{L}_{\text {quartks }} \ni \int d^{4} x\left(\mu \sum_{i=\text { quarks }} q_{i}^{\dagger} q_{i}+\mu_{5} \sum_{i=\text { quarks }} q_{i}^{\dagger} \gamma^{5} q_{i}\right)
$$

The chiral anomaly implies that the the corresponding chiral current density $J^{5 \mu}$ is not conserved, but its divergence is given by the so-called axial anomaly $[68,69]$, which in the case of interest is restricted only to include electromagnetic terms with Maxwell field strength $F_{\mu \nu}=\partial_{\mu} A_{\nu}-\partial_{\nu} A_{\mu}$ (with $A_{\mu}$ denoting the $\mathrm{U}(1)$ gauge potential, corresponding to the photon field), and its dual $\widetilde{F}_{\mu v}=\frac{1}{2} \epsilon_{\mu v \alpha \beta} F^{\alpha \beta}$ (with $\epsilon^{0123}=+1$ in our conventions):

$$
\partial_{\mu} J^{5 \mu}=\frac{e^{2}}{8 \pi^{2}} F_{\mu v} \widetilde{F}^{\mu v}=\frac{e^{2}}{2 \pi^{2}} \vec{E} \cdot \overrightarrow{\mathcal{B}},
$$

6 A chiral chemical potential has different values of the chemical potential for left and right chiral spinors. 
where $e$ is the electron charge, and $\vec{E}(\overrightarrow{\mathcal{B}})$ is the electric (magnetic) field, respectively, which is taken to be external in our discussion.

Integrating over three-space, we may rewrite Equation (25) in terms of the rate of change of the chirality $N_{5}=N_{R}-N_{L}[65]$ :

$$
\mu_{5} \frac{d N_{5}}{d t}=\frac{e^{2} \mu_{5}}{2 \pi^{2}} \int d^{3} x \vec{E} \cdot \overrightarrow{\mathcal{B}}
$$

In arriving at Equation (26), we take into account that the chiral chemical potential $\mu_{5}$ is the energy required to change a left-handed fermion into a right-handed one, which equivalently is the energy required to move a particle from the left-handed Fermi surface and place it onto the right-handed one. If $\mu_{L(R)}=\mu \mp \mu_{5}$ denotes the corresponding chemical potentials of the left (right) handed fermions, the above process costs an energy [65] $\mu_{R}-\mu_{L}=2 \mu_{5}$, and this will change the chirality $N_{5}$ by 2 . For an infinitesimal change $d N_{5}$ of the chirality then, the corresponding cost in energy is given by $\mu_{5} d N_{5}$, whose rate is then given by Equation (26). Conservation of energy implies that this amount must be compensated by the power of the electric field present in the system, which in terms of the electric current density $\vec{j}_{E}$ is provided by $\int d^{3} x \overrightarrow{j_{E}} \cdot \vec{E}$, thereby leading (on account of Equation (26)) to:

$$
\int d^{3} x \vec{j}_{E} \cdot \vec{E}=\mu_{5} \frac{d N_{5}}{d t}=\frac{e^{2} \mu_{5}}{2 \pi^{2}} \int d^{3} x \vec{E} \cdot \overrightarrow{\mathcal{B}}
$$

from which the CME follows [65], namely the existence of an electrical current proportional to the magnetic field strength and $\mu_{5}$ :

$$
\vec{J}_{E}=\frac{e^{2} \mu_{5}}{2 \pi^{2}} \int d^{3} x \overrightarrow{\mathcal{B}}=\frac{2 \alpha}{\pi} \mu_{5} \int d^{3} x \overrightarrow{\mathcal{B}},
$$

where the appearance of the fine structure constant $\alpha=e^{2} / 4 \pi$ as a proportionality factor indicates the quantum nature of the phenomenon, consistent with its origin from the chiral anomaly $[68,69]$. In addition, there is an induced chiral current, which is proportional to the chemical potential $\mu[65,72]$ :

$$
\vec{J}^{5}=\frac{e \mu}{2 \pi^{2}} \int d^{3} x \overrightarrow{\mathcal{B}}
$$

These effects have been defined in several independent ways in [65], including finite temperature $T \neq 0$ formulations, and thus it is argued that CME is independent of temperature for $T$-independent $\mu_{5}$ and $\mu$. Such an effect might have important phenomenological implications for the QG physics [65].

\subsection{Non-Contribution of the KR Background to the Chiral Magnetic Effect}

In string effective actions [52-54,58], the interpretation of the (totally antisymmetric) $H$-field as torsion is valid in an expansion in powers of the Regge slope $\alpha^{\prime}$ to first order (quartic in derivatives). For our cosmological case, a torsionful curved space-time is relevant; in such a space-time, any chiral anomaly that might characterise our system will also involve the generalised Riemann curvature tensor $\bar{R}_{\mu v \rho \sigma}(\bar{\omega})$ and its dual [58]

7 We note that this is the complete form of the anomaly in our case, which is characterised by the conservation of the H-torsion charge (Equation (17)), at a quantum level. Indeed, as discussed in [73], for a generic torsion three form $\mathbf{T}=\mathbf{d} \mathbf{e}^{a}+\bar{\omega}_{b}^{a} \wedge \mathbf{e}^{b}=\mathbf{K}_{b}^{a} \wedge \mathbf{e}^{b}$, with $\mathbf{e}^{a}$ the vielbein one-form and $\mathbf{K}$ the contorsion tensor, the righ- hand side of the anomaly (30) also contains the Nieh-Yan topological invariant density [74], $\mathcal{N}=\mathcal{N}=\mathbf{T}^{a} \wedge \mathbf{T}_{a}-\overline{\mathbf{R}}(\bar{\omega})_{a b} \wedge \mathbf{e}^{a} \wedge \mathbf{e}^{b}=\mathbf{d}\left(\mathbf{e}^{a} \wedge \mathbf{T}_{a}\right)$. In our case, $\star \mathbf{H} \propto \mathbf{e}_{a} \wedge \mathbf{T}^{a}$, and thus the Nieh-Yan invariant $\mathcal{N}$ vanishes identically on account of the torsion charge conservation constraint (Equation (17)), which can be written as $0=\mathbf{d} \star \mathbf{H}=\mathbf{d}\left(\mathbf{e}_{a} \wedge \mathbf{T}^{a}\right)=\mathcal{N}$. 


$$
\nabla_{\mu} J^{5 \mu}=\frac{e^{2}}{8 \pi^{2}} F_{\mu \nu} \widetilde{F}^{\mu v}-\frac{1}{192 \pi^{2}} \bar{R}_{\mu v \alpha \beta}(\bar{\omega}) \widetilde{\bar{R}}^{\mu \nu \alpha \beta}(\bar{\omega}) \equiv \mathcal{G}(A, \bar{\omega}),
$$

where the overline over a quantity denotes the presence of torsion, and $\bar{\omega}=\omega+H$ denotes (schematically) the torsionful connection, with $\omega$ the torsion-free connection and $H$ the KR field strength, which plays the role of (totally antisymmetric) torsion. The quantity $\nabla_{\mu}$ denotes the gravitational covariant derivative, with respect to the torsion-free connection ${ }^{8}$. Tensor duals are defined as $\widetilde{F}_{\mu \nu}=\frac{1}{2} \sqrt{-g} \varepsilon_{\mu \nu \rho \sigma} F^{\rho \sigma}$, and $\widetilde{R}_{\alpha \beta \mu v}=\frac{1}{2} \sqrt{-g} \varepsilon_{\mu \nu \rho \sigma} \bar{R}_{\alpha \beta}^{\rho \sigma}$, where $g$ is the determinant of the torsion-free metric corresponding to a (torsion-free) Riemann curvature tensor $R_{\mu \nu \rho \sigma}$.

The gravitational part of the anomaly (in differential form notation) is given by

$$
\left.\operatorname{Tr}(\overline{\mathbf{R}}(\bar{\omega}) \wedge \overline{\mathbf{R}}(\bar{\omega}))=\operatorname{Tr}(\mathbf{R}(\omega) \wedge \mathbf{R}(\omega))+\mathbf{d}\left[\operatorname{Tr}(\mathbf{H} \wedge \mathbf{R})+\mathbf{H} \wedge \mathbf{D} \mathbf{H}+\frac{2}{3} \mathbf{H} \wedge \mathbf{H} \wedge \mathbf{H}\right)\right],
$$

where $\mathbf{d}$ is the exterior derivative, $\mathbf{D}$ denotes the (torsion-free) gravitational covariant exterior form, $\mathbf{D} \mathbf{V}^{a}=\mathbf{d V}^{a}+\omega_{b}^{a} \wedge \mathbf{V}^{b}$, and the trace $\operatorname{Tr}$ is taken over tangent space (Latin) indices $a, b, \ldots$, i.e., $\operatorname{Tr}(\overline{\mathbf{R}}(\bar{\omega}) \wedge \overline{\mathbf{R}}(\bar{\omega}))=\overline{\mathbf{R}}_{b}^{a}(\bar{\omega}) \wedge \overline{\mathbf{R}}_{a}^{b}(\bar{\omega})$, with $\overline{\mathbf{R}}_{b}^{a}(\bar{\omega})=\frac{1}{2} \bar{R}_{\mu v b}{ }^{a} d x^{\mu} \wedge d x^{v}=\mathbf{d} \bar{\omega}_{b}^{a}+\bar{\omega}_{c}^{a} \wedge \bar{\omega}_{b}^{c}$, etc., where the indices $a, b, c \ldots$ are raised and lowered by the Minkowski metric. On the other hand, to maintain the conventional $U(1)$ gauge invariance, the Maxwell field strength is defined as in standard electrodynamics [58], with respect to the usual derivative (i.e., $\mathbf{F}=\mathbf{d A}$, obeying the Bianchi identity $\mathrm{dF}=0)$.

It is well known [70] that the torsion contributions (Equation (31)) to the anomaly (Equation (30)) may be removed by the addition of local renormalisation counterterms to the effective action, provided that the chiral current couples to a gauge field, which is the case of interest here. The anomaly becomes dependent only on the torsion-free spin connection $\omega^{9}$,

$$
\nabla_{\mu} J^{5 \mu}=\frac{e^{2}}{8 \pi^{2}} F_{\mu \nu} \widetilde{F}^{\mu \nu}-\frac{1}{192 \pi^{2}} R_{\mu \nu \alpha \beta}(\omega) \widetilde{R}^{\mu \nu \alpha \beta}(\omega) \equiv \mathcal{G}(A, \omega) .
$$

This implies a specific form of the low-energy effective action, which we restrict our attention to in this work. Indeed, as shown in [58], and discussed briefly in Section 1, imposing the constraint on the conservation of the torsion charge (Equation (17)) is equivalent to the addition of specific counterterms, which leads to the dual effective action (Equation (18)) in terms of the (Lagrange multiplier) KR-axion field $b(x)$. Thus, a QED effective action (in the concrete case the fermions are charged under electromagnetism) in a space-time with a torsionful connection, is equivalent to a QED action (Equation (18)) in a space time without torsion but with a dynamical KR axion field. The latter contains a dimension six four-fermion operator and a dimension five operator that couples the derivative of the $b$-field to the axial fermion current. By partial integration, then, this dimension-five term in the effective lagrangian yields a coupling of the $b$ field to the anomaly $\mathcal{G}(A, \omega)$ (32) without torsion. Since the torsion contributions to the anomaly are removable by an appropriate choice of counterterms, one should not expect any contribution of $B_{0}$ to the CME, which as discussed above (cf., Equations (26) and (28)), is linked to the chiral anomaly. Indeed, $B_{0}$ as an axial background, is known not to contribute to CME [66,67], as we now review briefly, for completeness.

\footnotetext{
The reader should notice that there is no $H$-torsion contribution to the covariant four-divergence of a four-vector.

As already mentioned, this can also be understood from the fact [70,71] that the chiral anomaly is associated with the index of the Dirac operator for fermions, and the latter is not affected by torsion, as it is associated with the topological quantity $\int_{\mathcal{M}} \operatorname{Tr}(R(\omega) \wedge R(\omega))=\int_{\mathcal{M}} \operatorname{Tr}(\bar{R}(\bar{\omega}) \wedge \bar{R}(\bar{\omega}))$, where $\mathcal{M}$ is a compact target-space-time manifold without boundary, and we took into account that the torsion contributions to the gravitational part of the anomaly (Equation (31)) is a total differential. The latter property would also imply that one can appropriately redefine the axial current by such torsion dependent terms [75,76], to arrive at a new gauge and Lorentz-invariant axial current, whose anomaly equation is torsion free.
} 
To this end, we first remark that, for a Robertson-Walker (RW) cosmological backgound, the $R \widetilde{R}$ term in Equation (32) vanishes identically ${ }^{10}$. Hence, for cosmological RW space-times, the axial anomaly is determined only by its gauge-field part. One can therefore discuss the CME in our context by a straightforward extension of the flat space-time case.

It suffices for our purposes to restrict our attention to a local frame, where the expansion of the universe can be ignored. (This would be the case if one were interested in examining the effects of $B_{0}$ on CME during the leptogenesis era, or in a quark-gluon (QG) plasma $[65,66]$ or in a neutron star $[79,80]$.) In the absence of an explicit $\mu_{5}$ term, we observe from the effective action (Equation (18)) that, at least formally, the background field $B_{0}=\dot{\bar{b}}$ seems to play a role analogous to a (generally temperature dependent) chiral chemical potential. If one adds a chemical potential $\mu_{5}$ term (e.g., to capture effects local in space-time in a QG plasma [65]), this will appear in the effective action for fermions as the combination

$$
\mu_{5}^{\mathrm{eff}} \equiv \mu_{5}-B_{0}(T),
$$

which has the apparent form of an effective chemical potential. One would thus naively expect a CME (Equation (28)), with $\mu_{5}$ replaced by $\mu_{5}^{\text {eff }}$ (33).

However, as argued in $[66,67]$, using different methods, the axial vector potential $B_{0}$ does not contribute to CME, and instead one has Equation (28), even if $B_{0} \neq 0$ is present ${ }^{11}$. The subtlety lies in the fact that, in the presence of a background field $B_{0}$, as we discussed in [39-41], the dispersion relations for the fermions are affected non-trivially by the presence of $B_{0}$, which differentiates it from the chiral chemical potential case; moreover, there are subtleties related to the order of taking the massless limit $m \rightarrow 0$. In the presence of a chiral chemical potential, an external constant magnetic field and a (generic, but constant) axial background (of which our (constant) $B_{0}$ is a special case), the CME is discussed in [67], within the framework of relativistic quantum mechanics [82]. It turns out to be important to take the massless (chiral fermion) limit $m \rightarrow 0$ at the end of the computation: one should assume massive fermions, in the presence of a $B_{0} \neq 0$, solve the corresponding Dirac equation, and only at the end take the limit $m \rightarrow 0$. Had one started, instead, with the chiral Lagrangian for massless quarks and then turned on an external vector time-like potential, $B_{0} \neq 0$, the appearance of $B_{0}$ contributions to the CME (through Equation (33)) would have occurred, however this would not be correct from the point of view of energy conservation [66].

The above results invalidate any arguments $[79,80]$ in favour of the axial background playing a role in the generation of instabilities and thus magnification of the magnetic fields in neutron stars. However the KR torsion might play a non-trivial role in the dynamo equation for the generation of magnetic fields $[83,84]$, and thus affect their strength independent of the CME. We hope to come back to a discussion of such effects in a future work.

10 In fact, it is only the gravitational-wave type fluctuations that contribute to the (torsion-free) Riemann-curvature-dependent part of the anomaly (Equation (30)), which can then lead to interesting scenarios for leptogenesis, different from our approach here [77]. Moreover, graviton fluctuations in the $R(\omega) \widetilde{R}(\omega)$ gravitational parts of the anomaly (Equation (30)) might play an important role in radiative Majorana mass generation for the right-handed neutrinos [78], as we review in Section 5.

11 We should stress that both the present and previous works of ours [41] pertain to cases in which the axial background is an independent field, e.g., the KR axion. We do not discuss here situations where the totally antisymmetric torsion is a chiral condensate of fermions. In such cases, as we remarked in [41], the free-fermion analysis of [67] needs to be modified to take proper account of the fermion self-interactions in Equation (18). For a discussion along those lines, and the potential role (in the early universe) of torsion arising from thermal condensates of massless chiral fermions, the reader is referred to Ref. [81]. 


\subsection{The Irrelevancy of Chern-Simons Terms for the Chiral Magnetic Effect}

In string theory, anomaly cancellation arguments necessitate [50,51] the modification of the three form representing the field strength of the Kalb-Ramond antisymmetric tensor by gauge (Yang-Mills (Y)) and gravitational (Lorentz (L)) Chern-Simons three forms $\Omega_{3}$

$$
\mathbf{H}=\mathbf{d B}+\frac{\alpha^{\prime}}{8 \kappa}\left(\Omega_{3 Y}-\Omega_{3 \mathrm{~L}}\right),
$$

such that the Bianchi identity (Equation (10)) is now modified to:

$$
\mathbf{d} \star \mathbf{H}=\frac{\alpha^{\prime}}{8 \kappa}[\operatorname{Tr}(\mathbf{F} \wedge \mathbf{F})-\operatorname{Tr}(\mathbf{R}(\omega) \wedge \mathbf{R}(\omega))],
$$

where now the trace is taken over appropriate gauge and Lorentz indices. This quantity is non-zero if there is no anomaly cancellation. As discussed above, by adding appropriate local counterterms to the effective action, one may arrange to add torsion contributions to the right-hand side of Equation (35), which will appear in a generalised curvature two-form, $\overline{\mathbf{R}}(\bar{\omega})$, inside the connection $\bar{\omega}=\omega+\mathbf{K}$, with $\mathrm{K}$ the contorsion. Equivalently, if one starts (in some effective field theory) from an anomaly equation with torsion, the latter can be removed via the above procedure. Thus, unless the gauge and gravitational anomalies are cancelled, the H-torsion charge (Equation (35)) is not conserved in the presence of Chern-Simons terms.

In our previous discussion, it was important that we added counterterms to the effective action of string-inspired QED with KR axions, in order to conserve the KR torsion charge in the quantum theory, via the constraint in Equation (17) in the path integral; this led to the absence of torsion from the effective theory (Equation (18)), the associated anomaly equation and to zero contributions of the KR axion to the CME. We now argue that the latter result is still valid even in the presence of Chern-Simons terms (Equation (35)).

To this end, it is convenient to use a simplified model, where only a $U(1)$ (electromagnetic gauge group) Chern-Simons (CS) term is present. This model is considered in [85,86], as a string-inspired prototype of a parity-violating version of quantum electrodynamics with torsion. For our purposes in the current work, we restrict ourselves to flat Minkowski space-times, since the introduction of space-time curvature does not affect our conclusions. Let us therefore consider, in the spirit of $[85,86]$, the following model $^{12}$ :

$$
\begin{aligned}
\mathcal{S}_{\mathrm{PV}} & =\int d^{4} x\left(-\frac{1}{4} F_{\mu \nu} F^{\mu \nu}+\frac{1}{2} \widetilde{H}_{\mu v \rho} \widetilde{H}^{\mu \nu \rho}\right) \\
& +\int d^{4} \bar{\psi}\left(\gamma^{\mu}\left(\imath \partial_{\mu}+q_{e} A_{\mu}\right)-m\right) \psi-\int d^{4} x B_{\mu} \bar{\psi} \gamma^{5} \gamma^{\mu} \psi+\ldots
\end{aligned}
$$

where $\widetilde{H}_{\mu v \rho}=\frac{1}{\kappa} \partial_{[\mu} B_{v \rho]}+\kappa A_{[\mu} F_{v \rho]}$, with $A_{\mu}$ an electromagnetic $U(1)$ field, $F_{\mu v}$ its Maxwell field strength, and in accordance to our previous notation, we work with dimensionless $B_{\mu v}$. Here, the $\mathrm{H}$-torsion has dimensions of $\left[\mathrm{mass}^{2}\right]$, to make contact with the conventions of $[85,86]$. The quantity $B_{\mu}$ is the axial torsion pseudovector

$$
B_{\mu}=\epsilon_{\mu \nu \rho \sigma} \widetilde{H}^{v \rho \sigma}
$$

12 This model can be obtained from a string-inspired effective theory of the modified Kalb-Ramond $H$-field with $U(1)$ CS terms, truncated to quadratic order in a derivative expansion, in a curved space-time with (non-duynamical) torsion $T_{\mu v \rho}$, which appears in both the Riemann curvature and the fermion gravitational covariant derivative, and is coupled to the $H_{\mu v \rho}$ field via $\int d^{4} x \sqrt{-g} \kappa^{-1} H_{\mu v \rho} T^{\mu v \rho}$ terms in the action $[85,86]$. This auxiliary torsion field can then be integrated exactly in the path integral, with the result that it obeys the constraint $T_{\mu \nu \rho}=\kappa H_{\mu \nu \rho}$. Substituting back to the action, and taking the Minkowski flat space-time limit, leads then to the action (Equation (36)). 
with $\epsilon_{\mu v \rho \sigma}$ the Levi-Civita antisymmetric symbol of flat Minkowski space-time, and arises from identifying the $\widetilde{H}_{\mu v \rho}$ as a (totally antisymmetric) torsion, which thus affects the connection in the gravitationally covariant derivative of the fermion field. In Equation (36), the ellipsis indicate the repulsive four fermion terms of the form of Equation (18), characterising every quantum torsion model, when the torsion is integrated out in a path-integral; such terms are suppressed by the Planck mass, and so are ignored for our low-energy analysis. The difference of the model (Equation (36)) from that considered in $[85,86]$ lies in the fact that here we explicitly consider the charged fermions $\psi$.

The (dimensionless) KR axion $b(x)$ field, is defined in this model from the dual of the $H_{\mu v \rho} \equiv$ $\kappa^{-1} \partial_{[\mu} B_{v \rho]}$ term in four space-time dimensions, $H_{\mu v \rho}=\kappa^{-1} \epsilon_{\mu v \rho \sigma} \partial^{\sigma} b(x)$. In our treatment in previous sections, the latter is identified with the Lagrange multiplier field implementing the path-integral constraint (Equation (17)) on the conservation of the torsion charge at quantum level [58]. Such a conservation is spoiled in the specific effective theory (Equation (36)), due to the Chern-Simons terms. Of course, by adding appropriate local counterterms to the effective action (Equation (36)), one can guarantee the conservation of torsion charge, in which case we are led back to the effective theory (Equation (18)), where there are no torsion contributions to the CME. Nonetheless, for the general reader, it will be instructive to verify explicitly this result in the presence of the Chern-Simons terms, which naively spoil the torsion-charge conservation.

To this end, one first observes from the action in Equation (36) that, from leading order in the inverse Planck-mass suppression factor $\kappa$, the axial torsion vector can be approximated by

$$
B_{\mu} \propto \partial_{\mu} b+\mathcal{O}(\kappa)
$$

As per our previous considerations, we consider a background $b \rightarrow \bar{b}$, in which the KR axion is linear in (cosmic) time, which yields $\bar{H}_{i j k}=\epsilon_{i j k 0} \dot{\bar{b}}=$ constant as the only non-zero components of $H_{\mu v \rho}$. This also implies that only the temporal component $B_{0}$ of the background pseudovector (Equation (38)) is non-vanishing and constant.

We are now well equipped to proceed with a study of the CME. It is instructive to follow the energy conservation arguments of [66]: we consider the rate of change of the total energy of the system (Equation (36)), that is of the sum of the energy density of the electromagnetic field and of the charged fermions in interaction with the external magnetic fields. We restrict ourselves to flat space-times. To this end, we first note from Equation (36) that the parity-violating interactions of the electromagnetic field with the KR axion are of the form,

$$
\mathcal{S}_{\mathrm{PV}} \ni \int d^{4} x \kappa H^{\mu \nu \rho} A_{[\mu} F_{v \rho]}=\int d^{4} x \epsilon^{\mu v \rho \sigma} \partial_{\sigma} b A_{[\mu} F_{v \rho]} .
$$

One might plausibly reason that, in the background $\dot{\bar{b}} \equiv B_{0}=$ constant, the terms in Equation (39) would yield a CME-like effect, with the current being proportional to $B_{0}$. In the presence of an external magnetic field $\overrightarrow{\mathcal{B}}$ in that case, one would obtain the parity-violating action terms ${ }^{13}$ : $\mathcal{S}_{\mathrm{PV}} \ni-\int d^{4} x B_{0} \epsilon^{i j k} A_{i} F_{j k}=-2 \int d^{4} x B_{0} \vec{A} \cdot \overrightarrow{\mathcal{B}}$. However, this naive expectation is not correct, as we now explain.

The parity-violating interaction terms in Equation (39) contribute to the energy density of the electromagnetic field, which, from leading order in $\kappa$ and for the constant $\dot{\bar{b}} \mathrm{KR}$ axion background, assumes the form:

$$
\mathcal{E}_{\text {em }}=\frac{1}{2}\left(\vec{E}^{2}+\overrightarrow{\mathcal{B}}^{2}\right)+2 \dot{\bar{b}} \vec{A} \cdot \overrightarrow{\mathcal{B}}
$$

The first term in parenthesis on the right-hand side is the standard Maxwell electrodynamics term.

13 The overall minus sign is due to the fact that the three-dimensional Levi-Civita symbol $\epsilon^{i j k}$ is defined as: $\epsilon^{i j k}=\epsilon^{0 i j k}=-\epsilon^{i j k 0}$. 
In the context of the conventional CME [65], we consider homogeneous electric $\vec{E}$ and magnetic $\overrightarrow{\mathcal{B}}$ fields, and in fact a constant intensity of the magnetic field $\vec{B}$. Under these conditions, the rate of change of the energy density $\mathcal{E}_{\text {em }}$ (Equation (40)) is:

$$
\frac{d}{d t} \mathcal{E}_{e m}=\vec{E} \cdot \frac{d \vec{E}}{d t}+2 B_{0} \vec{E} \cdot \overrightarrow{\mathcal{B}}
$$

It is important that at this stage we consider temporal dependence of the electric field. Eventually, we consider $\vec{E} \rightarrow 0$, parallel to the externally applied magnetic field, which are the conditions in which the CME is conventionally discussed [65].

On the other hand, as mentioned above [66,67], the energy of charged fermions $\mathcal{E}_{\text {fermion }}$ interacting with a constant external magnetic field $\vec{B}$ is determined by means of the Landau levels, of which only the lowest $n=0$, contributes to the CME current. In the presence of a chemical potential $\mu_{5}$, the rate of the corresponding energy density is given by

$$
\frac{d}{d t} \mathcal{E}_{\text {fermion }}=\frac{2 \alpha}{\pi} \mu_{5} \vec{E} \cdot \overrightarrow{\mathcal{B}},
$$

and has its origin in the chiral anomaly $[65,66]$. Notice that the axial torsion background $B_{0}$ (Equation (38)), does not contribute to this energy, as already mentioned [66,67].

Energy conservation requires [66]:

$$
\frac{d}{d t} \mathcal{E}_{e m}+\frac{d}{d t} \mathcal{E}_{\text {fermion }}=0 .
$$

From the equations of motion of the electromagnetic potential $A_{\mu}$ obtained from the action (Equation (36)), in our case, the only non-trivial one reads:

$$
\frac{d}{d t} \vec{E}+2 B_{0} \overrightarrow{\mathcal{B}}=-\vec{j}_{q}
$$

where $\vec{j}_{q}=q_{e} \bar{\psi} \vec{\gamma} \psi$, is the charged fermion current (with the notation $\vec{\gamma}$ referring collectively to the spatial Dirac matrices).

On account of Equations (44), (43), (42) and (41), we obtain

$$
\left(\overrightarrow{j_{q}}-\frac{2 \alpha}{\pi} \mu_{5} \overrightarrow{\mathcal{B}}\right) \cdot \vec{E}=0,
$$

which for an infinitesimal electric field $\vec{E} \rightarrow 0$ parallel to the constant magnetic field, yields the standard CME effect (Equation (28)). Notice here that had one naively set $\vec{E}=0$ in the Maxwell equation (Equation (44)), one would have erroneously derived a CME-like current $\vec{j}_{q}=-2 B_{0} \overrightarrow{\mathcal{B}}$ induced by the torsion background. This would violate energy conservation. What is actually happening here is that the quantum fluctuations of the electric field produce a $d \vec{E} / d t \neq 0$, and yield contributions to both the kinetic and the potential energy of the electrons that cancel any effects of $B_{0}$ on the induced current, which thus depends only on the chemical potential (Equations (45) and (28)). This concludes the proof of our statement that the presence of the Chern-Simons terms (Equation (35)) does not alter the conclusion that the torsion cannot contribute to the chiral magnetic effect. More formally the torsion terms can be removed from the chiral anomaly by addition of appropriate local counterterms to the effective action $[70,71]$, and thus they cannot contribute to phenomena associated with the anomaly, such as the CME.

\section{KR Axions and Anomalous Generation of Majorana Mass for the Right-Handed Neutrinos}

In our discussion thus far on leptogenesis, we have assumed a bare mass $m_{N}$ for RHN (Equation (3)), without providing any discussion on its microscopic origin. In standard scenarios, 
such a mass scale is provided by the seesaw mechanism [23-27]. We now review an approach to mass generation of RHN [78] which constitutes a novel mechanism for neutrino mass generation beyond the conventional seesaw framework [23-27]. The mechanism involves quantum fluctuations of a KR axion and a quantum anomaly.

An important aspect of the coupling of the KR axion quantum field $b(x))$ to the fermionic matter in the action (Equation (18)) is its shift symmetry, characteristic of an axion field. By shifting the field $b(x)$ by a constant, $b(x) \rightarrow b(x)+c$, the action only changes by total derivative terms, such as $c R^{\mu v \rho \sigma} \widetilde{R}_{\mu \nu \rho \sigma}$ and $c F^{\mu v} \widetilde{F}_{\mu v}$. These terms are irrelevant for the equations of motion and the induced quantum dynamics, provided the fields fall off sufficiently fast to zero at space-time infinity. The scenario for the anomalous Majorana mass generation through torsion proposed in [78], which we review briefly below, consists of augmenting the effective action (Equation (18)) by terms that break such a shift symmetry.

To this end, we first couple the KR axion $b(x)$ to another pseudoscalar axion field $a(x)$. In string-inspired models, such pseudoscalar axion $a(x)$ may be provided by the string moduli $[87,88]$. The proposed coupling occurs through a mixing in the kinetic terms of the two fields. Specifically, we consider the action:

$$
\begin{aligned}
\mathcal{S}= & \int d^{4} x \sqrt{-g}\left[\frac{1}{2}\left(\partial_{\mu} b\right)^{2}+\frac{b(x)}{192 \pi^{2} f_{b}} R^{\mu v \rho \sigma} \widetilde{R}_{\mu \nu \rho \sigma}+\frac{1}{2 f_{b}^{2}} J_{\mu}^{5} J^{5^{\mu}}+\gamma\left(\partial_{\mu} b\right)\left(\partial^{\mu} a\right)+\frac{1}{2}\left(\partial_{\mu} a\right)^{2}\right. \\
& \left.-i y_{a} a(x)\left(\bar{\psi}_{R}^{C} \psi_{R}-\bar{\psi}_{R} \psi_{R}^{C}\right)\right]+\ldots, \quad f_{b}=\left(\frac{3 \kappa^{2}}{8}\right)^{-1 / 2}=\frac{M_{P}}{\sqrt{3 \pi}}
\end{aligned}
$$

where the ... indicate terms in the low-energy string effective action, including SM ones, that are not of direct relevance to our purposes in the present article. The anomaly equation (Equation (32)) has been used to yield the second term on the right hand side of Equation (46), by partial integration of the corresponding $\partial b(x)-J^{5}$ term of the action (Equation (18)). Here, we have ignored gauge fields, which are not of interest to us, given that there is no direct coupling of RHN to them. Moreover, for our purposes, the form of the axion $a(x)$ potential, including details of its mass $M_{a}$, are not relevant [78]. Above, $\psi_{R}^{C}$ is the charge-conjugate right-handed fermion $\psi_{R}$, with the corresponding four-component Majorana spinor being defined as $\psi=\psi_{R}+\left(\psi_{R}\right)^{C}$, and $\gamma$ is a real parameter to be constrained later on. The Yukawa coupling $y_{a}$ of the axion moduli field $a$ to right-handed sterile neutrino matter $\psi_{R}$ may be due to non perturbative effects (e.g., string instantons), which are known (along with the axion potential itself) to break the shift symmetry: $a \rightarrow a+c$.

It is convenient to diagonalize the axion kinetic terms in Equation (46) by redefining the KR axion field as follows: $b(x) \rightarrow b^{\prime}(x) \equiv b(x)+\gamma a(x)$. It can then be easily seen [78] that the $b^{\prime}$ field decouples and can be integrated out in the path integral, leaving behind an axion field $a(x)$ coupled both to matter fermions and to the operator $R^{\mu \nu \rho \sigma} \widetilde{R}_{\mu v \rho \sigma}$. As discussed in [78], however, the approach is only valid for $|\gamma|<1$, otherwise the axion field would appear as a ghost, i.e. with the wrong sign of its kinetic terms, which would indicate an instability of the model. This is the only restriction of the parameter $\gamma$. In this case, we may redefine the axion field to appear with a canonical normalised kinetic term, implying the effective action:

$$
\begin{aligned}
\mathcal{S}_{a}= & \int d^{4} x \sqrt{-g}\left[\frac{1}{2}\left(\partial_{\mu} a\right)^{2}-\frac{\gamma a(x)}{192 \pi^{2} f_{b} \sqrt{1-\gamma^{2}}} R^{\mu \nu \rho \sigma} \widetilde{R}_{\mu \nu \rho \sigma}\right. \\
& \left.-i \frac{y_{a}}{\sqrt{1-\gamma^{2}}} a(x)\left(\bar{\psi}_{R}^{C} \psi_{R}-\bar{\psi}_{R} \psi_{R}^{C}\right)+\frac{1}{2 f_{b}^{2}} J_{\mu}^{5} J^{5 \mu}\right] .
\end{aligned}
$$

Evidently, the action $\mathcal{S}_{a}$ in Equation (47) corresponds to a canonically normalised axion field $a(x)$, coupled both to the curvature of space-time, à la torsion, with a modified coupling $\gamma /\left(192 \pi^{2} f_{b} \sqrt{1-\gamma^{2}}\right)$, and to fermionic matter with chirality-changing Yukawa-like couplings of the form $y_{a} / \sqrt{1-\gamma^{2}}$.

The mechanism for the anomalous Majorana mass generation is shown in Figure 1. Only graviton fluctuations and axion $a(x)$ fields couple to the RHN at leading orders. We may estimate the 
two-loop Majorana mass for the RHN, $M_{R}^{N}$, in quantum gravity with an effective UV energy cut-off $\Lambda$, by adopting the effective field-theory framework of [89]:

$$
M_{R}^{N} \sim \frac{\sqrt{3} y_{a} \gamma \kappa^{5} \Lambda^{6}}{49152 \sqrt{8} \pi^{4}\left(1-\gamma^{2}\right)} .
$$

In a UV complete theory such as strings, the cutoff $\Lambda$ and the Planck mass scale $M_{P}$ are related.

If the cut-off $\Lambda$ is of the same order as the reduced Planck mass of the four-dimensional theory, i.e., $\kappa \Lambda \sim 1$, then, from Equation (48), we observe that for $y_{a} \gamma=\mathcal{O}\left(10^{-6}\right)$, one obtains $M_{R}^{N} \sim 10^{5} \mathrm{GeV}$, of the order of the RHN in our leptogenesis scenario. On the other hand, for much lower $\Lambda$ of order of the GUT scale, $\Lambda \sim 10^{16} \mathrm{GeV}$, one obtains RHN masses $M_{R}^{N}=\mathcal{O}(16) \mathrm{keV}$, i.e., in the warm dark matter regime of the $v \mathrm{MSM}[21,22]$, consistent with current stringent constraints [90,91]. The mass hierarchy among the RHN is arranged by appropriate choices of the Yukawa couplings $y_{a}, a=1,2,3$, in that case.

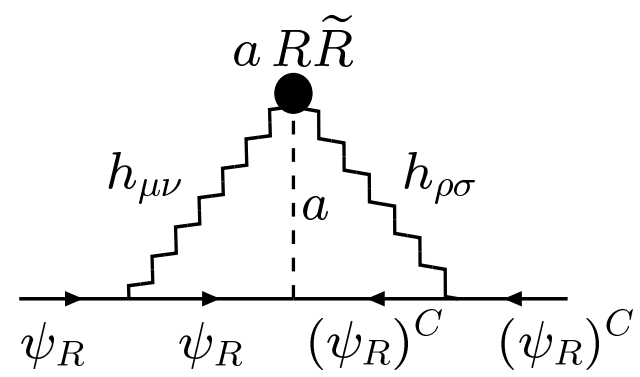

Figure 1. Two-loop Feynman graph giving rise to anomalous generation of Majorana mass for the right-handed neutrinos $\psi_{R}$ [78]. The black circle denotes the operator $a(x) R_{\mu \nu \lambda \rho} \widetilde{R}^{\mu \nu \lambda \rho}$ induced by anomalies (at one-loop). The fields $h_{\mu \nu}$ (wavy lines) denote graviton fluctuations. Straight lines with arrows denote right handed neutrino fields and their conjugates.

We now remark that in string theory there are several axion-like (pseudoscalar) fields $a_{i}(x)$, $i=1,2, \ldots, n$, originating from flux fields that exist in the spectrum $[87,88]$, in addition to the aforementioned $B_{\mu \nu}$ Kalb-Ramond field. One can then assume [78] the existence of Yukawa couplings with right-handed neutrinos, provided some non-perturbative instanton effects are responsible for a breaking of the shift symmetry. These string-theory axion fields could mix with each other. Such a mixing can give rise to reduced UV sensitivity of the two-loop graph shown in Figure 1. To make this point explicit, let us consider a scenario with $n$ axion fields, $a_{1,2, \ldots, n}$, of which only $a_{1}$ has a kinetic mixing term $\gamma$ with the KR axion $b$ and only $a_{n}$ has a Yukawa coupling $y_{a}$ to right-handed neutrinos $\psi_{R}$. The other axions $a_{2,3, \ldots, n}$ have a next-to-neighbour mixing pattern. In such a model, the kinetic terms of the effective action are given by

$$
\mathcal{S}_{a}^{\text {kin }}=\int d^{4} x \sqrt{-g}\left[\frac{1}{2} \sum_{i=1}^{n}\left(\left(\partial_{\mu} a_{i}\right)^{2}-M_{i}^{2}\right)+\gamma\left(\partial_{\mu} b\right)\left(\partial^{\mu} a_{1}\right)-\frac{1}{2} \sum_{i=1}^{n-1} \delta M_{i, i+1}^{2} a_{i} a_{i+1}\right],
$$

where the mixing mass terms $\delta M_{i, i+1}^{2}$ are constrained to be $\delta M_{i, i+1}^{2}<M_{i} M_{i+1}$, to obtain a stable positive mass spectrum for all axions. As a consequence of the next-to-nearest-neighbour mixing, the UV behaviour of the off-shell transition $a_{1} \rightarrow a_{n}$, described by the propagator matrix element $\Delta_{a_{1} a_{n}}(p)$, changes drastically, i.e., $\Delta_{a_{1} a_{n}}(p) \propto 1 /\left(p^{2}\right)^{n} \sim 1 / E^{2 n}$. Assuming for simplicity that all axion masses and mixings are equal, i.e., $M_{i}^{2}=M_{a}^{2}$ and $\delta M_{i, i+1}^{2}=\delta M_{a}^{2}$, the anomalously generated Majorana mass may be estimated to be

$$
M_{R}^{N} \sim \frac{\sqrt{3} y_{a} \gamma \kappa^{5} \Lambda^{6-2 n}\left(\delta M_{a}^{2}\right)^{n}}{49152 \sqrt{8} \pi^{4}\left(1-\gamma^{2}\right)},
$$

for $n \leq 3$, and

$$
M_{R}^{N} \sim \frac{\sqrt{3} y_{a} \gamma \kappa^{5}\left(\delta M_{a}^{2}\right)^{3}}{49152 \sqrt{8} \pi^{4}\left(1-\gamma^{2}\right)} \frac{\left(\delta M_{a}^{2}\right)^{n-3}}{\left(M_{a}^{2}\right)^{n-3}}
$$


for $n>3$. It is then not difficult to see that three axions $a_{1,2,3}$ with next-to-neighbour mixing as discussed above would be sufficient to obtain a UV finite (cut-off- $\Lambda$-independent) result for $M_{R}^{N}$ at the two-loop level. Of course, beyond the two loops, $M_{R}$ will depend on higher powers of the energy cut-off $\Lambda$, i.e., $\Lambda^{n>6}$, but if $\kappa \Lambda \ll 1$, these higher-order effects are expected to be subdominant.

In the above $n$-axion-mixing scenarios, we note that the anomalously generated Majorana mass term will only depend on the mass-mixing parameters $\delta M_{a}^{2}$ of the axion fields and not on their masses themselves, as long as $n \leq 3$. Instead, for axion-mixing scenarios with $n>3$, the induced Majorana neutrino masses are proportional to the factor $\left(\delta M_{a}^{2} / M_{a}^{2}\right)^{n}$, which gives rise to an additional suppression for heavy axions with masses $M_{a} \gg \delta M_{a}$.

\section{Conclusions}

In this work, we have reviewed our previous studies of leptogenesis induced by a (rather generic) LV and CPTV time-like axial background $B_{0}$, which is provided by the field strength of the antisymmetric tensor KR field appearing in the massless spectrum of microscopic, ultraviolet complete, string-inspired models. We discuss briefly scenarios where the background $B_{0}$ is either constant, for a given epoch of the universe, or varying slowly with the temperature of the early universe. The phenomenology of leptogenesis, associated with a lepton asymmetry generated by the (asymmetric) decays of heavy right-handed Majorana neutrinos (RHN) into SM leptons and antileptons, in the presence of temperature-dependent axial backgrounds, remains largely unchanged from the constant background case, and is consistent with the stringent current-era epoch constraints on LV and CPTV. Fine tuning however is required to ensure the suppression of the vacuum energy density. This can be provided by bulk anti-de-Sitter contributions to the vacuum energy density of our universe in brane models, where our world is viewed as a three brane propagating in a higher dimensional bulk space time.

As a byproduct of our analysis, we also argue that the background $B_{0}$ does not contribute to the so-called Chiral Magnetic Effect (CME), i.e., the induction of an electrical current proportional to external magnetic fields, which characterises physical systems in the presence of chiral chemical potentials, $\mu_{5}$ (i.e., where there is a non-zero difference, $\mu_{L}-\mu_{R} \neq 0$, between the chemical potentials of left- and right-handed chiral fermions). Since, during the leptogenesis era, one may have encountered primordial magnetic fields, and given the apparent role of the background $B_{0}$ in the effective Lagrangian as a dynamical contribution to $\mu_{5}$, it is natural to ask whether $B_{0}$ contributes to CME. As we argued above, this is not the case. This result can be understood either from the point of view of a generic axial background, which is known for energetic reasons not to contribute to CME, or of our string-inspired model, from the fact that the background $B_{0}$ is associated with the (totally antisymmetric) "H-torsion" induced by the KR field. The CME is associated with the chiral anomaly. In addition, it is a well known that in string effective theories the $\mathrm{H}$-torsion contributions to the anomaly can be removed by a choice of the renormalisation scheme. Thus, the null contribution of the $\mathrm{H}$-torsion to the CME should be expected on these grounds.

Finally, the role of quantum fluctuations of the KR axion in generating an anomalous Majorana mass for the RHN themselves is also described. For this latter scenario, a kinetic mixing between the KR axion with ordinary axion fields $a(x)$ (QCD or string inspired) is assumed, together with shift-symmetry breaking chirality-changing Yukawa interactions of the axions $a(x)$ with the Majorana neutrinos (which might be the result of non-perturbative effects (instantons) in microscopic string models). This mechanism is novel and goes beyond the conventional seesaw. It is interesting to remark that, within our string framework, it is known that there can be several axion fields, which allows an ultraviolet-cutoff independent RHN Majorana mass for the special case of three axion fields and three RHNs.

It would be interesting to pursue further the detailed phenomenology and cosmology of such models, in particular to examine the effects of temperature in the induced RHN mass, and thus embed fully this mechanism within our CPTV leptogenesis scenario. This will be investigated in a future work. 
Funding: This research was funded in part by STFC (UK) research grant ST/P000258/1.

Acknowledgments: The authors wish to thank the organisers of the International Conference of New Frontiers in Physics 2018 for organising such high-level and stimulating event. N.E.M. wishes to thank the organisers for their kind invitation to give a plenary talk. He also acknowledges a scientific associateship ("Doctor Vinculado") at IFIC-CSIC-Valencia University (Spain).

Conflicts of Interest: The authors declare no conflict of interest. The funders had no role in the design of the study; in the collection, analyses, or interpretation of data; in the writing of the manuscript, or in the decision to publish the results.

\section{References}

1. Kuzmin, V.A.; Rubakov, V.A.; Shaposhnikov, M.E. On the Anomalous Electroweak Baryon Number Nonconservation in the Early Universe. Phys. Lett. 1985, 155B, 36. [CrossRef]

2. Gavela, M.B.; Hernandez, P.; Orloff, J.; Pene, O. Standard model CP violation and baryon asymmetry. Mod. Phys. Lett. A 1994, 9, 795. [CrossRef]

3. Gavela, M.B.; Hernandez, P.; Orloff, J.; Pene, O.; Quimbay, C. Standard model CP violation and baryon asymmetry. Part 2: Finite temperature. Nucl. Phys. B 1994, 430, 382. [CrossRef]

4. Ade, P.A.R.; Aghanim, N.; Armitage-Caplan, C.; Arnaud, M.; Ashdown, M.; Atrio-Barandela, F.; Aumont, J.; Baccigalupi, C.; Banday, A.J.; Barreiro, R.B.; et al. Planck 2013 results. XVI. Cosmological parameters. Astron. Astrophys. 2014, 571, A16.

5. Spergel, D.N.; Verde, L.; Peiris, H.V.; Komatsu, E.; Nolta, M.R.; Bennett, C.L.; Halpern, M.; Hinshaw, G.; Jarosik, N.; Kogut, A.; et al. First year Wilkinson Microwave Anisotropy Probe (WMAP) observations: Determination of cosmological parameters. Astrophys. J. Suppl. 2003, 148, 175. [CrossRef]

6. Sakharov, A.D. Violation of CP Invariance, C Asymmetry, and Baryon Asymmetry of the Universe. Sov. Phys. Usp. 1991, 34, 392. [CrossRef]

7. Buchmuller, W.; di Bari, P.; Plumacher, M. Leptogenesis for pedestrians. Ann. Phys. 2005, 315, 305. [CrossRef]

8. Davidson, S.; Nardi, E.; Nir, Y. Leptogenesis. Phys. Rep. 2008, 466, 105. [CrossRef]

9. Pilaftsis, A. The Little Review on Leptogenesis. J. Phys. Conf. Ser. 2009, 171, 012017. [CrossRef]

10. Pilaftsis, A. Advances in Leptogenesis. J. Phys. Conf. Ser. 2013, 447, 012007. [CrossRef]

11. Biondini, S.; Bodeker, D.; Brambilla, N.; Garny, M.; Ghiglieri, J.; Hohenegger, A.; Laine, M.; Mendizabal, S.; Millington, P.; Salvio, A.; et al. Status of rates and rate equations for thermal leptogenesis. Int. J. Mod. Phys. A 2018, 33, 1842004. [CrossRef]

12. Cohen, A.G.; Kaplan, D.B.; Nelson, A.E. Progress in electroweak baryogenesis. Ann. Rev. Nucl. Part. Sci. 1993, 43, 27. [CrossRef]

13. Trodden, M. Electroweak baryogenesis. Rev. Mod. Phys. 1999, 71, 1463. [CrossRef]

14. Riotto, A.; Trodden, M. Recent progress in baryogenesis. Ann. Rev. Nucl. Part. Sci. 1999, 49, 35. [CrossRef]

15. Buchmuller, W. Baryogenesis: 40 Years Later. arXiv 2007, arXiv:0710.5857.

16. Fukugita, M.; Yanagida, T. Baryogenesis without Grand Unification. Phys. Lett. B 1986, 174, 45. [CrossRef]

17. Luty, M.A. Baryogenesis via leptogenesis. Phys. Rev. D 1992, 45, 455. [CrossRef]

18. Pilaftsis, A. CP violation and baryogenesis due to heavy Majorana neutrinos. Phys. Rev. D 1997, 56, 5431.[CrossRef]

19. Buchmuller, W.; Peccei, R.D.; Yanagida, T. Leptogenesis as the origin of matter. Ann. Rev. Nucl. Part. Sci. 2005, 55, 311. [CrossRef]

20. Strumia, A. Baryogenesis via leptogenesis. In Particle Physics Beyond the Standard Model, Proceedings, Summer School on Theoretical Physics, 84th Session, Les Houches, France, 1-26 August 2005; Kazakov, D., Lavignac, S., Dalibard, J., Eds.; Elsevier: Amsterdam, The Netherlands, 2006.

21. Shaposhnikov, M.; Tkachev, I. The nuMSM, inflation, and dark matter. Phys. Lett. B 2006, 639, 414. [CrossRef]

22. Shaposhnikov, M. Neutrino masses, dark matter, baryon asymmetry and inflation can be explained at once. Subnucl. Ser. 2011, 47, 167.[CrossRef]

23. Minkowski, P. $\mu \rightarrow e \gamma$ at a rate of one out of 109 muon decays? Phys. Lett. B 1977, 67, 421. [CrossRef]

24. Gell-Mann, M.; Ramond, P.; Slansky, R. Supergravity; Freedman, D.Z., van Nieuwenhuizen, P., Eds.; North-Holland: Amsterdam, The Netherlands, 1979.

25. Yanagida, T. Horizontal gauge symmetry and masses of neutrinos. In Proceedings of the Workshop on the Unified Theory and the Baryon Number in the Universe, Tsukuba, Japan, 13-14 February 1979. 
26. Sawada, O.; Sugamoto, A.; Mohapatra, R.N.; Senjanovic, G. Neutrino Mass and Spontaneous Parity Violation. Phys. Rev. Lett. 1980, 44, 912.

27. Schechter, J.; Valle, J.W.F. Neutrino Masses in SU(2) $\times$ U(1) Theories. Phys. Rev. D 1980, 22, 2227. [CrossRef]

28. Gonzalez-Garcia, M.C.; Maltoni, M. Phenomenology with Massive Neutrinos. Phys. Rep. 2008, 460, 1. [CrossRef]

29. Forero, D.V.; Tortola, M.; Valle, J.W.F. Global status of neutrino oscillation parameters after Neutrino-2012. Phys. Rev. D 2012, 86, 073012. [CrossRef]

30. Streater, R.F.; Wightman, A.S. PCT, Spin and Statistics, and All That; Princeton University Press: Princeton, NJ, USA, 2000; 207p.

31. Chaichian, M.; Dolgov, A.D.; Novikov, V.A.; Tureanu, A. CPT Violation Does Not Lead to Violation of Lorentz Invariance and Vice Versa. Phys. Lett. B 2011, 699, 177. [CrossRef]

32. Chaichian, M.; Fujikawa, K.; Tureanu, A. Lorentz invariant CPT violation. Eur. Phys. J. C 2013, 73, 2349. [CrossRef]

33. Arraut, I. The Quantum Yang Baxter conditions and the dispersion relations for the Nambu-Goldstone bosons. arXiv 2016, arXiv:1611.05035.

34. Arraut, I. Spontaneous symmetry breaking as a triangular relation between pairs of Goldstone bosons and the degenerate vacuum: Interactions of D-branes. arXiv 2017, arXiv:1705.02218.

35. Arraut, I. The origin of the mass of the Nambu?Goldstone bosons. Int. J. Mod. Phys. A 2018, 33, 1850041. [CrossRef]

36. Greenberg, O.W. CPT violation implies violation of Lorentz invariance. Phys. Rev. Lett. 2002, 89, 231602. [CrossRef]

37. Mavromatos, N.E.; Sarkar, S. CPT-Violating Leptogenesis induced by Gravitational Defects. Eur. Phys. J. C 2013, 73, 2359. [CrossRef]

38. Ellis, J.; Mavromatos, N.E.; Sarkar, S. Environmental CPT Violation in an Expanding Universe in String Theory. Phys. Lett. B 2013, 725, 407. [CrossRef]

39. de Cesare, M.; Mavromatos, N.E.; Sarkar, S. On the possibility of tree-level leptogenesis from Kalb? Ramond torsion background. Eur. Phys. J. C 2015, 75, 514. [CrossRef] [PubMed]

40. Bossingham, T.; Mavromatos, N.E.; Sarkar, S. Leptogenesis from Heavy Right-Handed Neutrinos in CPT Violating Backgrounds. Eur. Phys. J. C 2018, 78, 113. [CrossRef]

41. Bossingham, T.; Mavromatos, N.E.; Sarkar, S. The role of temperature dependent string-inspired CPT violating backgrounds in leptogenesis and the chiral magnetic effect. arXiv 2018, arXiv:1810.13384.

42. Colladay, D.; Kostelecky, V.A. Lorentz violating extension of the standard model. Phys. Rev. D 1998, 58, 116002. [CrossRef]

43. Kostelecky, V.A.; Russell, N. Data Tables for Lorentz and CPT Violation. Rev. Mod. Phys. 2011, 83, 11. [CrossRef]

44. Mavromatos, N.E. Theory overview of testing fundamental symmetries. Hyperfine Interact. 2014, $228,7$. [CrossRef]

45. Kostelecky, V.A.; Mewes, M. Lorentz-violating electrodynamics and the cosmic microwave background. Phys. Rev. Lett. 2007, 99, 011601. [CrossRef] [PubMed]

46. Das, M.; Mohanty, S.; Prasanna, A.R. Constraints on background torsion from birefringence of CMB polarization. Int. J. Mod. Phys. D 2013, 22, 1350011. [CrossRef]

47. Bertolami, O.; Colladay, D.; Kostelecky, V.A.; Potting, R. CPT violation and baryogenesis. Phys. Lett. B 1997, 395, 178. [CrossRef]

48. Dolgov, A.D. CPT violation and particle-antiparticle asymmetry in cosmology. Phys. Atom. Nucl. 2010, 73, 588. [CrossRef]

49. Ulmer, S.; Smorra, C.; Mooser, A.; Franke, K.; Nagahama, H.; Schneider, G.; Higuchi, T.; van Gorp, S.; Blaum, K.; Matsuda, Y.; et al. High-precision comparison of the antiproton-to-proton charge-to-mass ratio. Nature 2015, 524, 196. [CrossRef]

50. Green, M.B.; Schwarz, J.H.; Witten, E. Superstring Theory. Vol. 1: Introduction. In Cambridge Monographs on Mathematical Physics; Cambridge University Press: Cambridge, UK, 1987; 469p.

51. Superstring Theory. Vol. 2: Loop Amplitudes, Anomalies and Phenomenology. In Cambridge Monographs on Mathematical Physics; Cambridge University Press: Cambridge, UK, 1987; 596p. 
52. Gross, D.J.; Sloan, J.H. The Quartic Effective Action for the Heterotic String. Nucl. Phys. B 1987, $291,41$. [CrossRef]

53. Metsaev, R.R.; Tseytlin, A.A. Order alpha-prime (Two Loop) Equivalence of the String Equations of Motion and the Sigma Model Weyl Invariance Conditions: Dependence on the Dilaton and the Antisymmetric Tensor. Nucl. Phys. B 1987, 293, 385. [CrossRef]

54. Bento, M.C.; Mavromatos, N.E. Ambiguities in the Low-energy Effective Actions of String Theories with the Inclusion of Antisymmetric Tensor and Dilaton Fields. Phys. Lett. B 1987, 190, 105. [CrossRef]

55. Antoniadis, I.; Bachas, C.; Ellis, J.R.; Nanopoulos, D.V. An Expanding Universe in String Theory. Nucl. Phys. B 1989, 328, 117. [CrossRef]

56. Mavromatos, N.E.; Rizos, J. Exact solutions and the cosmological constant problem in dilatonic domain wall higher curvature string gravity. Int. J. Mod. Phys. A 2003, 18, 57. [CrossRef]

57. Mavromatos, N.E. String inspired higher curvature terms and the Randall-Sundrum scenario. Phys. Rev. D 2000, 62, 124004. [CrossRef]

58. Duncan, M.J.; Kaloper, N.; Olive, K.A. Axion hair and dynamical torsion from anomalies. Nucl. Phys. B 1992, 387, 215. [CrossRef]

59. Greenberg, O.W. Why is CPT fundamental? Found. Phys. 2006, 36, 1535. [CrossRef]

60. Ade, P.A.R.; Aghanim, N.; Arnaud, M.; Ashdown, M.; Aumont, J.; Baccigalupi, C.; Banday, A.J.; Barreiro, R.B.; Bartlett, G.; Bartolo, N.; et al. Planck 2015 results. XIII. Cosmological parameters. Astron. Astrophys. 2016, 594, A13. [CrossRef]

61. Cota, R.; Barranco, J.; Delepine, D.; Khalil, S. Time-dependent Lepton Asymmetry with Primordial Magnetic Fields. PoS DSU 2012, 2012, 026, doi:10.22323/1.161.0026. [CrossRef]

62. Cota, R. Lepton Asymmetry with Primordial Magnetic Fields. J. Phys. Conf. Ser. 2013, 468, 012003. [CrossRef]

63. Dvornikov, M.; Semikoz, V.B. Leptogenesis via hypermagnetic fields and baryon asymmetry. J. Cosmol. Astropart. Phys. 2012, 2012, 040; Erratum in 2012; 2012, E01. [CrossRef]

64. Long, A.J.; Sabancilar, E.; Vachaspati, T. Leptogenesis and Primordial Magnetic Fields. J. Cosmol. Astropart. Phys. 2014, 2014, 036. [CrossRef]

65. Fukushima, K.; Kharzeev, D.E.; Warringa, H.J. The Chiral Magnetic Effect. Phys. Rev. D 2008, 78, 074033. [CrossRef]

66. Kaplan, D.B.; Reddy, S.; Sen, S. Energy Conservation and the Chiral Magnetic Effect. Phys. Rev. D 2017, 96, 016008. [CrossRef]

67. Dvornikov, M. Chiral magnetic effect in the presence of an external axial-vector field. Phys. Rev. D 2018, 98, 036016. [CrossRef]

68. Adler, S.L. Axial-Vector Vertex in Spinor Electrodynamics. Phys. Rev. 1969, 177, 2246. [CrossRef]

69. Bell, J.S.; Jackiw, R. A PCAC puzzle: $\pi^{0} \rightarrow \gamma \gamma$ in the $\sigma$-model. Nuovo Cim. A 1969, 60, 47.

70. Hull, C.M. Anomalies, Ambiguities and Superstrings. Phys. Lett. 1986, 167B, 51. [CrossRef]

71. Mavromatos, N.E. A Note on the Atiyah-singer Index Theorem for Manifolds With Totally Antisymmetric $H$ Torsion. J. Phys. A 1988, 21, 2279. [CrossRef]

72. Metlitski, M.A.; Zhitnitsky, A.R. Anomalous axion interactions and topological currents in dense matter. Phys. Rev. D 2005, 72, 045011. [CrossRef]

73. Chandia, O.; Zanelli, J. Reply to "Comment on 'Topological invariants, instantons, and the chiral anomaly on spaces with torsion"'. Phys. Rev. D 2001, 63, 048502. [CrossRef]

74. Nieh, H.T.; Yan, M.L. Some comments on finite subgroups of SU(3). J. Math. Phys. 1982, 23, 373. [CrossRef]

75. Dobado, A.; Maroto, A.L. The lepton anomaly in the presence of torsion. Phys. Rev. D 1996, 54, 5185. [CrossRef]

76. Dvoeglazov, V.V. Photon and Poincare Group; Nova Science Publishers, Inc.: Hauppauge, NY, USA, 1999; pp. 298-312.

77. Alexander, S.H.S.; Peskin, M.E.; Sheikh-Jabbari, M.M. Leptogenesis from gravity waves in models of inflation. Phys. Rev. Lett. 2006, 96, 081301. [CrossRef]

78. Mavromatos, N.E.; Pilaftsis, A. Anomalous Majorana Neutrino Masses from Torsionful Quantum Gravity. Phys. Rev. D 2012, 86, 124038. [CrossRef]

79. Dvornikov, M. Impossibility of the strong magnetic fields generation in an electron-positron plasma. Phys. Rev. D 2014, 90, 041702. [CrossRef] 
80. Dvornikov, M.; Semikoz, V.B. Magnetic field instability in a neutron star driven by the electroweak electron-nucleon interaction versus the chiral magnetic effect. Phys. Rev. D 2015, 91, 061301. [CrossRef]

81. Dolan, B.P. Chiral fermions and torsion in the early Universe. Class. Quant. Grav. 2010, 27, 095010; Erratum in Class. Quant. Grav. 2010, 27, 249801. [CrossRef]

82. Balantsev, I.A.; Popov, Y.V.; Studenikin, A.I. On the problem of relativistic particles motion in strong magnetic field and dense matter. J. Phys. A 2011, 44, 255301. [CrossRef]

83. Garcia de Andrade, L.C. Cosmological and astrophysical consequences from the magnetic dynamo equation in torsioned spacetime and teleparallel gravity. Class. Quant. Grav. 2016, 33, 025006. [CrossRef]

84. Garcia de Andrade, L.C. Metric-torsion decay of non-adiabatic chiral helical magnetic fields against chiral dynamo action in bouncing cosmological models. Eur. Phys. J. C 2018, 78, 530. [CrossRef]

85. Majumdar, P.; SenGupta, S. Parity violating gravitational coupling of electromagnetic fields. Class. Quant. Grav.1999, 16, L89. [CrossRef]

86. Kar, S.; Majumdar, P.; SenGupta, S.; Sur, S. Cosmic optical activity from an inhomogeneous Kalb-Ramond field. Class. Quant. Grav. 2002, 19, 677. [CrossRef]

87. Arvanitaki, A.; Dimopoulos, S.; Dubovsky, S.; Kaloper, N.; March-Russell, J. String Axiverse. Phys. Rev. D 2010, 81, 123530. [CrossRef]

88. Cicoli, M.; Goodsell, M.; Ringwald, A. The type IIB string axiverse and its low-energy phenomenology. J. High Energy Phys. 2012, 2012, 146. [CrossRef]

89. Donoghue, J.F. General relativity as an effective field theory: The leading quantum corrections. Phys. Rev. D 1994, 50, 3874. [CrossRef]

90. Perez, K.; Ng, K.C.Y.; Beacom, J.F.; Hersh, C.; Horiuchi, S.; Krivonos, R. Almost closing the nuMSM sterile neutrino dark matter window with NuSTAR. Phys. Rev. D 2017, 95, 123002. [CrossRef]

91. Yunis, R.; Argüelles, C.R.; Mavromatos, N.E.; Moliné, A.; Krut, A.; Rueda, J.A.; Ruffini, R. New constraints on sterile neutrino dark matter from the Galactic Center. arXiv 2018, arXiv:1810.05756.

(C) 2018 by the authors. Licensee MDPI, Basel, Switzerland. This article is an open access article distributed under the terms and conditions of the Creative Commons Attribution (CC BY) license (http:/ / creativecommons.org/licenses/by/4.0/). 